\title{
Effects of Neutron Irradiations in Various Crystal Samples of Large Size for Future Crystal Calorimeter
}

Liyuan Zhang, Member, IEEE, Rihua Mao, Member, IEEE, and Ren-Yuan Zhu, Senior Member, IEEE

\begin{abstract}
In this paper, we report an investigation on the radiation damage effects induced by neutrons in large size crystal scintillator: BGO, CeF 3 , LYSO:Ce and PWO. The irradiations were carried out by using fast neutrons from one ${ }^{241} \mathrm{Am}-\mathrm{Be}$ and two ${ }^{252} \mathrm{Cf}$ sources. The optical and scintillation properties of these samples, including UV excitation and emission spectra, longitudinal transmission, light output, decay kinetics and light response uniformity, were measured before and after the irradiations. The neutron induced photo-current was also measured, and was used to estimate the readout noise under the neutron flux expected by an electromagnetic calorimeter at a very severe radiation environment. Because of its high light output and excellent radiation resistance LYSO:Ce crystal is found to have the smallest neutron induced readout noise as compared to other large size crystals, indicating it is a good candidate material for a future crystal calorimeter in a severe radiation environment.
\end{abstract}

\section{INTRODUCTION}

I NORGANIC crystal scintillators have played an important role in high energy physics detectors. Their use in future high energy physics experiments, however, faces a challenge to survive in a unprecedented severe radiation environment, such as the SLHC. To be used in such environment crystals must maintain its performance, such as light yield, transmission and readout noise. In this study, radiation damage effect of fast neutrons is investigated for several crystal samples of large size. $4 \mathrm{MeV}$ neutrons from one ${ }^{241} \mathrm{Am}-\mathrm{Be}$ source and 2.5 $\mathrm{MeV}$ neutrons from two ${ }^{252} \mathrm{Cf}$ sources are used for irradiation source. Neutron induced radiation damage effects in these samples are measured.

\section{SAMPLES AND EXPERIMENTAL APPARATUS}

Fig. 1 is a photo showing six samples used in this study. They are, from top to bottom: a $\mathrm{CeF}_{3}$ sample with dimension of $3.0 \times 14 \times 3.0 \mathrm{~cm}^{3}$ from Shanghai Institute of Ceramics (SIC), a BGO sample with dimension of $2.5 \times 20 \times 2.5$ $\mathrm{cm}^{3}$ from SIC, two LYSO:Ce samples with dimension of $2.5 \times 20 \times 2.5 \mathrm{~cm}^{3}$ from Saint-Gobain Ceramics \& Plastics, Inc. (SG) and Sichuan Institute of Piezoelectric and Acousto-optic Technology (SIPAT) respectively and two PWO samples with dimension of $2.85 \times 22 \times 3.0 \mathrm{~cm}^{3}$ from Bogoroditsk TechnoChemical Plant (BTCP) and SIC respectively.

This work was supported in part by the U.S. Department of Energy under grant DE-FG03-92-ER-40701 and the U.S. National Science Foundation Award PHY-0612805.

The Authors are with the California Institute of Technology, Pasadena, CA 91125 USA (e-mail: zhu@hep.caltech.edu).

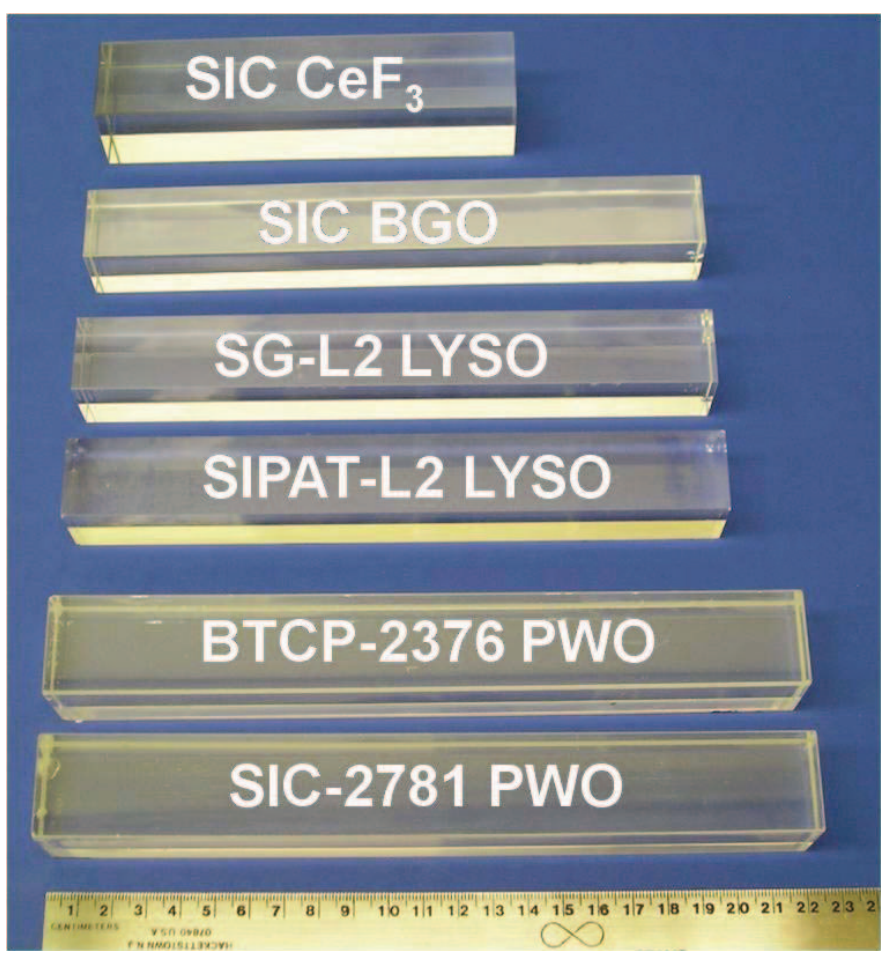

Fig. 1. A photo showing the six crystal samples used in this study.

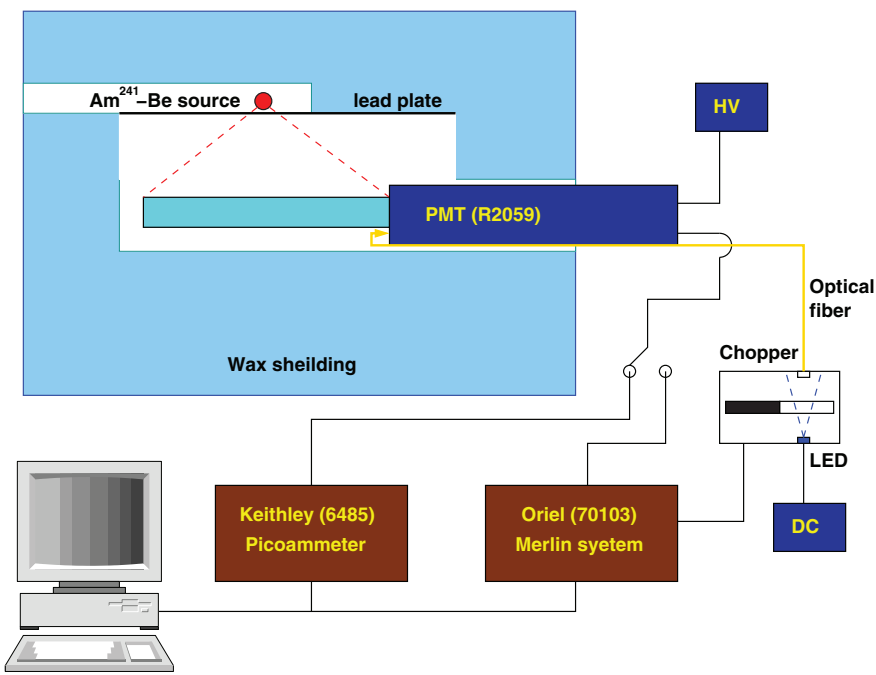

Fig. 2. The setup used for neutron irradiation by an ${ }^{241} \mathrm{Am}$-Be source and for the anode photo-current measurement. 

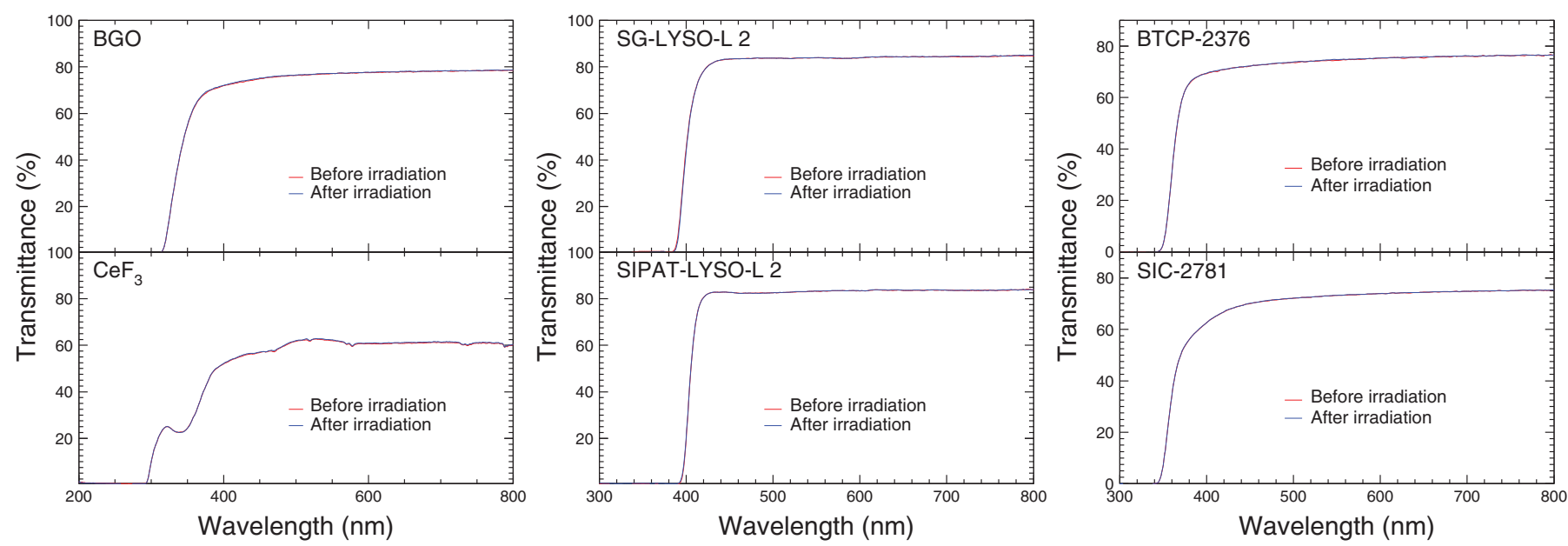

Fig. 3. The longitudinal transmittance measured before (red) and after (blue) the irradiations by $4 \mathrm{MeV}$ neutrons from the ${ }^{241} \mathrm{Am}-\mathrm{Be}$ source with an accumulated fluence of $6 \times 10^{8} \mathrm{n} / \mathrm{cm}^{2}$ is shown as a function of wavelength for BGO and $\mathrm{CeF}_{3}$ (Left), two LYSO (Middle) and two PWO (Right) samples.
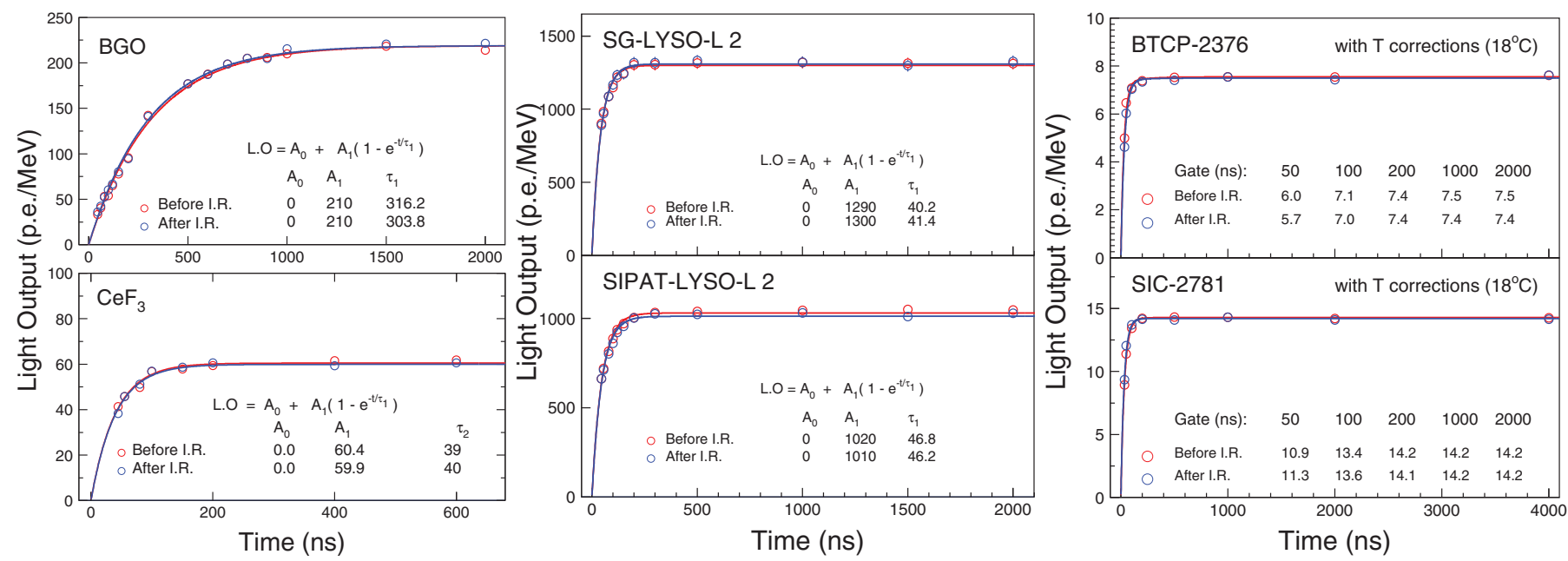

Fig. 4. The light output measured before (red) and after (blue) the irradiations by $4 \mathrm{MeV}$ neutrons from the ${ }^{241} \mathrm{Am}-\mathrm{Be}$ source with an accumulated fluence of $6 \times 10^{8} \mathrm{n} / \mathrm{cm}^{2}$ is shown as a function of the integration time for BGO and $\mathrm{CeF}_{3}$ (Left), two LYSO (Middle) and two PWO (Right) samples.

Fig. 2 shows the set-up used for the irradiations by an ${ }^{241} \mathrm{Am}-\mathrm{Be}$ source and for the photo-current measurement. The distance between the sample and the source was adjusted by trading off between the neutron flux and its uniformity along the crystal. The optimized distance was determined to be $14 \mathrm{~cm}$ and the average of the $4 \mathrm{MeV}$ neutron flux at the sample surface was about $10^{3} \mathrm{n} \mathrm{s}^{-1} \mathrm{~cm}^{-2}$ with a uniformity of better than $20 \%$ for $20 \mathrm{~cm}$ long samples. The sample was wrapped with the Tyvek paper except for one end which was coupled to a PMT (Hamamatsu R2059) with an air gap. The neutron induced photo-current was measured by a multimeter (Keithley Picoammeter 6485). The gain of the PMT was monitored by using a chopped blue LED light shooting to the PMT window through an optical fiber. The set-up for two ${ }^{252} \mathrm{Cf}$ sources is similar to Fig. 2 with the ${ }^{241} \mathrm{Am}-\mathrm{Be}$ source replaced by two ${ }^{252} \mathrm{Cf}$ sources separated by $15 \mathrm{~cm}$. The optimized distance from source to crystal was determined to be $8 \mathrm{~cm}$ and the average of the $2.5 \mathrm{MeV}$ neutron flux at the sample surface was $1.4 \times 10^{4} \mathrm{n} \mathrm{s}^{-1} \mathrm{~cm}^{-2}$ with a uniformity of better than $10 \%$ for $20 \mathrm{~cm}$ long samples. All samples were irradiated by the ${ }^{241} \mathrm{Am}$-Be source for about one week with accumulated fluence of $6 \times 10^{8} \mathrm{n} \mathrm{cm}^{-2}$. All samples are also irradiated by two ${ }^{252} \mathrm{Cf}$ sources for about one hour. Week long irradiations by two ${ }^{252} \mathrm{Cf}$ sources are being carried out for all samples.

\section{EFFECT ON OPTICAL AND SCINTILLATION PROPERTIES}

To investigate neutron induced effects, the optical and scintillation properties of these samples were measured before and after the irradiations. The UV excitation and emission spectra were measured by using a Hitachi F4500 fluorescence spectrophotometer. The longitudinal transmissions was measured by using a Perkin Elmer Lambda-950 spectrometer. The light output, decay kinetics and the light response uniformity were measured by using a Photonis XP2254b PMT through and a home-made DAQ system, of which the detailed information can be found in ref. [1].

The optical and scintillation properties of these samples, including UV excitation and emission spectra, longitudinal 

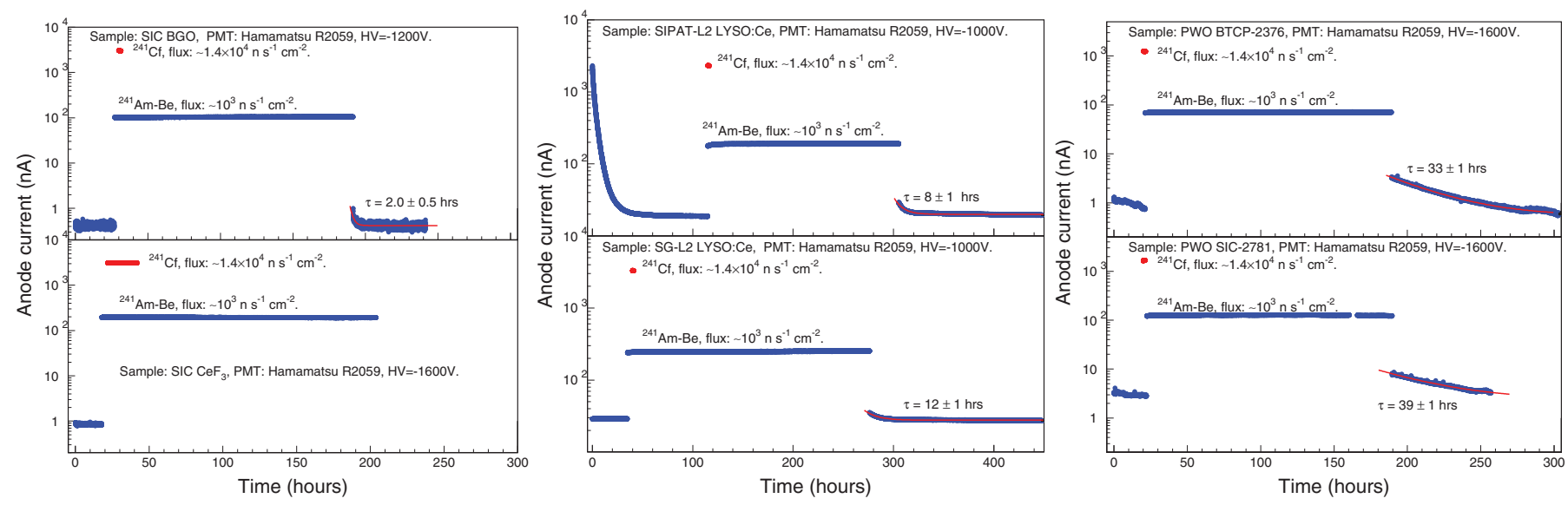

Fig. 5. The anode photo-current measured during irradiations by the ${ }^{241} \mathrm{Am}-\mathrm{Be}$ (blue dots) and ${ }^{252} \mathrm{Cf}$ sources (red dots) is shown as a function of time for BGO and $\mathrm{CeF}_{3}$ (Left), two LYSO (Middle) and two PWO (Right) samples. Also shown is the exponential fit of the cool-down time of the neutron-induced phosphorescence.

TABLE I

SUMMARY OF THE NEUTRON INDUCED READOUT NOISE

\begin{tabular}{c|c|c|c|c|c|c|c|c}
\hline \hline $\begin{array}{c}\text { Sample } \\
\text { ID }\end{array}$ & $\begin{array}{c}\text { Dimension } \\
\mathrm{cm}^{3}\end{array}$ & $\begin{array}{c}\text { L.Y. } \\
\text { p.e./MeV }\end{array}$ & $\begin{array}{c}\mathrm{F}^{a} \\
\text { p.e. } n^{-1} \mathrm{~cm}^{2}\end{array}$ & $\begin{array}{c}\mathrm{F}^{c} \\
\text { p.e. } \mathrm{n}^{-1} \mathrm{~cm}^{2}\end{array}$ & $\begin{array}{c}\sigma_{\text {bar }}^{a} \\
\mathrm{MeV}\end{array}$ & $\begin{array}{c}\sigma_{\text {bar }}^{c} \\
\mathrm{MeV}\end{array}$ & $\begin{array}{c}\sigma_{\text {end }}^{a} \\
\mathrm{MeV}\end{array}$ & $\begin{array}{c}\sigma_{\text {end }}^{c} \\
\mathrm{MeV}\end{array}$ \\
\hline \hline SIC-2781 PWO & $2.8 \times 22 . \times 3.0$ & 14.2 & $1.7 \times 10^{3}$ & $1.6 \times 10^{3}$ & 3.0 & 2.9 & 16.9 & 16.4 \\
BTCP-2376 PWO & $2.8 \times 22 . \times 3.0$ & 7.5 & $1.0 \times 10^{3}$ & $1.2 \times 10^{3}$ & 4.3 & 4.8 & 24.6 & 26.9 \\
\hline SIPAT-L2 LYSO & $2.5 \times 20 \times 2.5$ & 1940 & $2.6 \times 10^{5}$ & $2.3 \times 10^{3}$ & 0.4 & 0.4 & 2.2 & 2.0 \\
SG-L2 LYSO & $2.5 \times 20 . \times 2.5$ & 2480 & $3.4 \times 10^{3}$ & $3.2 \times 10^{3}$ & 0.3 & 0.3 & 1.9 & 1.9 \\
\hline SIC BGO & $2.5 \times 20 . \times 2.5$ & 340 & $2.0 \times 10^{4}$ & $4.1 \times 10^{4}$ & 1.4 & 1.9 & 7.7 & 11.0 \\
\hline SIC CeF & $3.0 \times 14 . \times 3.0$ & 60 & $2.6 \times 10^{3}$ & $3.0 \times 10^{3}$ & 1.2 & 1.3 & 7.0 & 7.5 \\
\hline \hline
\end{tabular}

transmission, light output, decay kinetics and light response uniformity, were measured before and after the week long neutron irradiation by the ${ }^{241} \mathrm{Am}$-Be source with an integrated fluence of $6 \times 10^{8} \mathrm{n} / \mathrm{cm}^{2}$. Figs. 3 and 4 show the longitudinal transmissions as a function of wavelength and the light outputs as a function of the integration time respectively for six samples. It is clear that the results obtained are consistent for all samples, indicating no evident damage induced by 4 $\mathrm{MeV}$ neutron irradiation with a cumulated fluence of $6 \times 10^{8}$ $\mathrm{n} \mathrm{cm}^{-2}$.

\section{Neutron Induced Photo-CurRent}

Fig. 5 shows the anode photo-current as a function of time, measured before, under and after the irradiation of the ${ }^{241} \mathrm{Am}$ Be source irradiation (blue dots) as well as under two ${ }^{252} \mathrm{Cf}$ sources (red dots), fox six samples. Since the LYSO samples have a strong phosphorescence if exposed to light they have to be kept in dark for more than $24 \mathrm{~h}$ before starting the test as shown in the middle top plot for the SIPAT-L2 sample. The reading of the neutron induced photo-currents during irradiations was set to be around hundred $\mathrm{nA}$ by adjusting the PMT bias to avoid possible PMT gain saturation. The photocurrent was found to be stable during the one week irradiations by the ${ }^{241} \mathrm{Am}-\mathrm{Be}$ source, indicating no cumulative effect from neutrons. Also shown in the figure is the time constant for the cool-down of the neutron induced phosphorescence. It is about 2, 10 and 35 hours for BGO, LYSO and PWO respectively. The corresponding time constant of $\mathrm{CeF}_{3}$ is yet to be determined.

\section{Neutron Induced Readout Noise}

By using the measured anode current, the neutron induced photo-electron coefficient (F) can be calculated for each sample, which is defined as the neutron induced photo-electron number under unit neutron flux:

$$
F=\frac{\frac{\text { photo-current }}{\text { Charge electron } \times \text { Gain } P M T}}{\text { Flux } x_{\text {crystal }}}\left(\text { p.e. } \mathrm{n}^{-1} \mathrm{~cm}^{2}\right) .
$$

Since the neutron flux is accompanied by undetermined $\gamma$-rays, which is not easy to be properly subtracted, the coefficient $F$ calculated here can be seen as a upper limit with contributions of undetermined $\gamma$-rays. By using this coefficient "F", the neutron induced photo-electron numbers in more severe environment can be calculated for a defined readout gate, which is defined as about 4 times of the corresponding scintillation decay time to fully integrate the scintillation light for each crystal. The corresponding readout gate used in this calculation is $100 \mathrm{~ns}$ for PWO, $200 \mathrm{~ns}$ for LYSO and $\mathrm{CeF}_{3}$ and $1 \mu \mathrm{s}$ for BGO. The fluctuation of this photo-electron number in this gate is the induced readout noise. At the LHC, the accumulated neutron fluence after 10 years or $5 \times 10^{7} \mathrm{~s}$ operation at peak luminosity are estimated to be up to $5.3 \times 10^{13} \mathrm{n} \mathrm{cm}^{-2}$ and $1.7 \times 10^{15} \mathrm{n} \mathrm{cm}^{-2}$ for the barrel and endcap respectively of the CMS ECAL [2]. We use these number and assume 10 times higher neutron fluxes for the SLHC, the photoelectron 
number in the readout gate for each sample can be calculated as

$$
Q=\frac{10 \times \text { Fluence }_{10 y r} \times \text { Gate }_{\text {readout }} \times F}{5.0 \times 10^{7}}(\text { p.e }),
$$

and the energy equivalent noise is calculated by using the standard deviation of photoelectron number:

$$
\sigma=\frac{\sqrt{Q}}{L O}(M e V) \text {. }
$$

Table I summarizes the coefficient " $\mathrm{F}$ " and corresponding readout noises at barrel $\left(\sigma_{b a r}\right)$ and endcap $\left(\sigma_{e n d}\right)$, where the superscript "a" represents the results from the measurements with the ${ }^{241} \mathrm{Am}-\mathrm{Be}$ source and "c" the results by using the ${ }^{252} \mathrm{Cf}$ sources. All samples, except the BGO sample, show consistent results obtained by using these two kind of sources. The coefficients "F" determined by using two sources show a difference by a factor of two for the BGO sample. Further work is needed to understand this discrepancy. The LYSO samples show the smallest readout noises because of their high light yield.

\section{Summary}

By using an ${ }^{241} \mathrm{Am}$-Be source and two ${ }^{252} \mathrm{Cf}$ sources fast neutron induced effects is investigated for large size BGO, $\mathrm{CeF}_{3}$, LYSO and PWO crystals samples. No variation was found in optical and scintillation properties for all samples after the irradiations by $4 \mathrm{MeV}$ neutrons from a ${ }^{241} \mathrm{Am}-\mathrm{Be}$ source with accumulated fluence of $6 \times 10^{8} \mathrm{n} \mathrm{cm}^{-2}$. Tests for higher neutron fluence is underway. The neutron induced photo-current during irradiation was measured and used to calculate the readout noise in a severe environment, such as like SLHC. The results show that the LYSO has the smallest neutron induced readout noise, indicating it is a good candidate for future crystal calorimeter in severe radiation environment.

\section{REFERENCES}

[1] R. Mao, L. Zhang and R.Y. Zhu, "Optical and Scintillation Properties of Inorganic Scintillators in High Energy Physics," IEEE Trans. Nucl. Sci., Vol.55, pp.2425-2430, 2008.

[2] The CMS Electromagnetic Calorimeter Project, CERN/LHCC 97-33 (1997). 